\title{
Compassionate extubation protocol to improve team communication and support in the neonatal intensive care unit
}

\author{
Kelsey Donoho $\mathbb{1}^{1} \cdot$ Mallory Fossa ${ }^{2,5} \cdot$ Sarah Dabagh $^{2,6} \cdot$ Menchie Caliboso $^{2,7} \cdot$ Debra Lotstein $^{2,3} \cdot$ Srikumar Nair $^{4}$
}

Received: 3 November 2020 / Revised: 20 April 2021 / Accepted: 29 April 2021 / Published online: 18 May 2021

(c) The Author(s), under exclusive licence to Springer Nature America, Inc. 2021

\begin{abstract}
Objective Compassionate extubation (CE) can be stressful for staff and families in the neonatal intensive care unit (NICU). Our quality improvement initiative developed and implemented a novel symptom management and family support checklist and post-debriefing template to improve team communication and staff support.

Study design An interprofessional team performed a needs assessment, determined key drivers and intervention steps, and implemented changes using Plan-Do-Study-Act cycles. Outcomes included nursing perception of good communication with the medical team, nursing assessment of patient comfort after CE, and frequency of post-event debrief. Outcomes were analyzed using time series design with 12 months baseline data and 6 months post-implementation monitoring.

Result Eighteen events were studied. Respondents endorsing "good" communication with the medical team increased by $60 \%$, and debrief participation rate improved by $96 \%$.

Conclusion Implementation of a CE checklist and post-event debriefing sheet was associated with increased rate of debriefs and improved team communication.
\end{abstract}

Supplementary information The online version contains supplementary material available at https://doi.org/10.1038/s41372021-01085-8.

Kelsey Donoho

kelseydonohomd@gmail.com

1 Division of Neonatology, Department of Pediatrics, LAC+USC Medical Center, Keck School of Medicine of the University of Southern California, Los Angeles, CA, USA

2 Division of Comfort and Palliative Care, Department of Anesthesia and Critical Care Medicine, Children's Hospital Los Angeles, Los Angeles, CA, USA

3 Department of Pediatrics, Keck School of Medicine of the University of Southern California, Los Angeles, CA, USA

4 Division of Neonatology, Department of Pediatrics, Fetal and Neonatal Institute, Children's Hospital Los Angeles, Keck School of Medicine of the University of Southern California, Los Angeles, CA, USA

5 Present address: Division of Pain and Palliative Medicine, Connecticut Children's Medical Center, Hartford, CT, USA

6 Present address: Department of Palliative Care, Memorial Care Miller Women and Children's Hospital, Long Beach, CA, USA

7 Present address: Department of Quality Information and Analytics, University of California, Los Angeles, Los Angeles, CA, USA

\section{Introduction}

Compassionate extubation (CE), the withdrawal of lifesustaining invasive ventilatory support, occurs frequently before infants die in the Newborn Intensive Care Unit (NICU); three-quarters of all NICU deaths in the United States occur during withdrawal of life-sustaining interventions [1-3]. CE can be a stressful experience for both families and staff in the NICU. Much of the existing literature on staff comfort with end-of-life care in the NICU represents the nursing population, though other staff members, such as physicians, respiratory therapists and social workers, are presumed to face many of the same challenges. For neonatal nurses who are simultaneously tasked with effectively managing symptom burden as well as providing support to the infant's family, there are multiple sources of stress during a CE [4]. Nurses in this setting may struggle with negative emotions including moral distress and feelings of professional inadequacy. This places them at risk for experiencing compassion fatigue, which in turn impairs their provision of effective family support during CE [5]. NICU parents are highly sensitive to providers' degree of empathy: their most common criticism surrounding their infant's death was a lack of sensitivity and emotional support from staff [6]. Conversely, a positive 
relationship between staff and parents can dramatically ease parents' grief process [7].

In past studies, NICU nurses report feeling unprepared, both educationally and emotionally, to support parents in end-of-life settings [8, 9]. This lack of confidence may reduce parental satisfaction with NICU care [10]. Neonatal and pediatric intensive care nurses also experience communication challenges during end-of-life care. Nurses report dissatisfaction with communication between nursing and the treating physician or team [11], as well as communication challenges with families [12].

Although CE is relatively common, no published consensus guideline exists to aid medical management and communication during $\mathrm{CE}$ in the NICU. Checklists have been helpful in improving consistency and quality in other complex, multi-step clinical processes [13], but no CE-specific checklist for the NICU exists in the medical literature. Studies have also demonstrated that formal and informal debriefing sessions are an important component of psychological coping for staff who provide end-of-life care $[4,14]$, yet institutional support is often unavailable $[11,15]$.

Lack of a standardized approach to patient care and staff support pertaining to CE was recognized in our NICU as a potential target for improvement, as there was no existing model or institutional guideline. To address this, we sought to create a standardized approach to $\mathrm{CE}$ in our NICU using the Model for Improvement [16] to guide our efforts. For our primary intervention, we focused on developing a CE checklist, with the intention that this tool would allow a time-efficient and thorough review of the plan of care, as well as a post-debriefing tool to encourage feedback and emotional processing. Nurses were chosen as our primary target for surveys given their extended time at bedside during these events. We assessed outcomes before and after protocolization, with the focused aim of improving team communication and nursing staff support during $\mathrm{CE}$ in our NICU. While we focused on NICU nurses in this study, we hope to generalize our findings and expand our efforts with the ultimate goal of increasing patient comfort and reducing distress for both families and interprofessional NICU staff.

\section{Methods}

\section{Setting}

This quality improvement project was conducted at a large, urban, free-standing pediatric academic medical center with a level IV, 58 bed NICU. Medical care for patients undergoing CE is provided by neonatology faculty and fellows, pediatric residents, and advanced practice providers, with the support of our institution's pediatric palliative care team on a case-by-case basis.

\section{Planning the intervention}

An interprofessional improvement team was formed by members of the neonatology and palliative care divisions. During the design phase, we reviewed the medical literature and considered other institutions' protocols to determine best practices. We performed retrospective chart reviews of patients undergoing CE in our NICU between October 1, 2017 and September 30, 2018. We included information on time from $\mathrm{CE}$ to death, and principal diagnosis contributing to re-direction of goals of care. Diagnosis was categorized as: severe neurological impairment, cardiopulmonary failure, multi-organ failure, or multiple congenital anomalies. A needs assessment survey evaluating perceptions of $\mathrm{CE}$ management and communication was distributed electronically to NICU nurses who responded anonymously. Using 5-point level-of-agreement Likert questions, nurses were asked to retrospectively rate patient comfort, nurse comfort, and quality of communication within the medical team.

We then reviewed the baseline data and institutional best practices. We outlined our pre-intervention NICU processes, identified key areas of improvement, and tested potential solutions using the Plan-Do-Study-Act (PDSA) model. Key areas for improvement included enhancing communication with family and nursing staff, developing guidelines for symptom management, prioritizing provider availability during and after $\mathrm{CE}$, and encouraging a standard post-extubation staff debrief.

For the first stage of intervention, the quality improvement team developed and implemented a checklist to be used by the medical team prior, during and after a CE. The checklist was based on the results of needs assessment as well as other institutional protocols (Supplementary Fig. 1). A debriefing template was also developed to guide discussion and facilitate adoption of post-CE staff debriefing (Supplementary Fig. 2). Within 1 week after each CE event, online post-event surveys were distributed to medical providers, nurses, and respiratory therapists who cared for patients who underwent $\mathrm{CE}$ as identified during chart review to examine the efficacy of the checklist and debrief template; in cases where staff were involved in multiple $\mathrm{CE}$ events, they were invited to complete a survey for each event.

As each event was reviewed, opportunities for improved awareness and ease of use were addressed, which defined the three PDSA cycles described in this paper: PDSA 1 began after the checklist and debriefing sheet were launched; PDSA 2 grouped the checklist and debriefing sheet together as a packet with an introductory cover to promote staff use; PDSA 3 began after both the checklist and 
Fig. 1 Key driver diagram. Driver diagram to increase percentage of nurses endorsing good communication within the medical team, increase percentage of nurses who found it easy to maintain their patient's comfort during and after the compassionate extubation, and increase the rate of post-event staff debriefs.

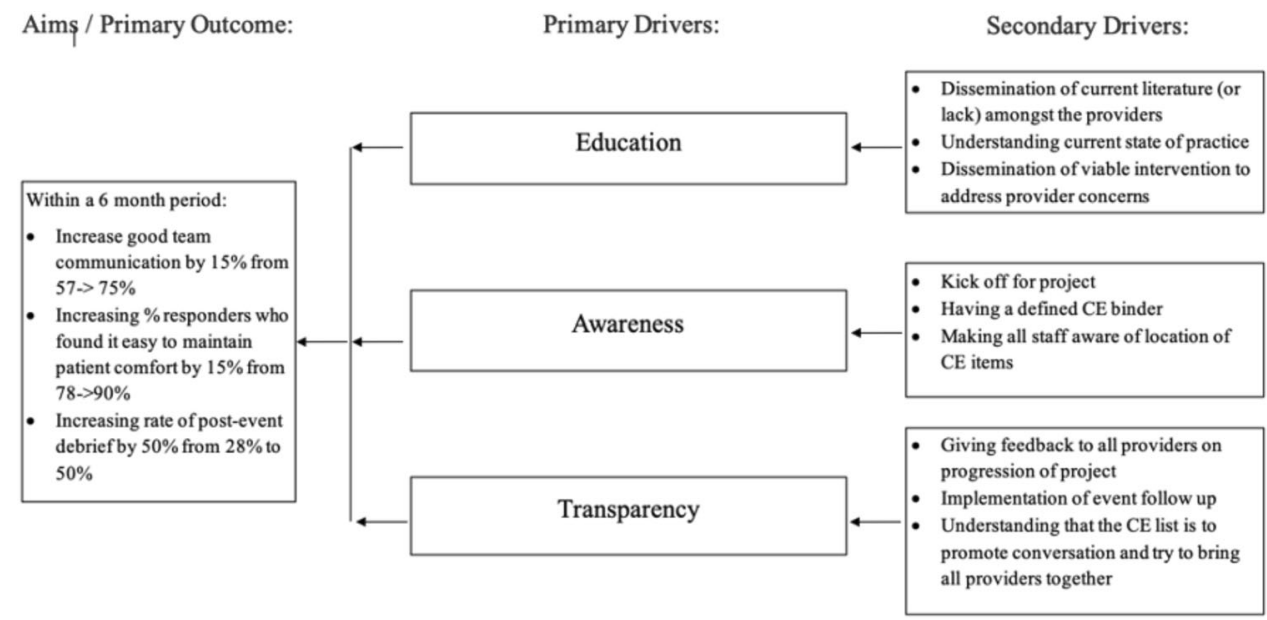

Table 1 Characteristics of baseline compassionate extubation (CE) events.

\begin{tabular}{lll}
\hline & $\begin{array}{l}\text { Baseline CE } \\
\text { events }(n=29)\end{array}$ & $\begin{array}{l}\text { Post-intervention CE } \\
\text { events }(n=18)\end{array}$ \\
\hline $\begin{array}{l}\text { Time (days, hours, minutes) } \\
\text { Average }\end{array}$ & $1 \mathrm{~d} 4 \mathrm{~h}$ & $6 \mathrm{~h} 3 \mathrm{~m}$ \\
Median & $1 \mathrm{~h} 2 \mathrm{~m}$ & $1 \mathrm{~h} 22 \mathrm{~m}$ \\
Range & $1 \mathrm{~m}-33 \mathrm{~d} 14 \mathrm{~h}$ & $18 \mathrm{~m}-2 \mathrm{~d} 21 \mathrm{~h} 38 \mathrm{~m}$ \\
$\begin{array}{l}\text { Indication for Re-Direction } \\
\text { of Care }\end{array}$ & \\
$\begin{array}{l}\text { Severe neurologic } \\
\text { impairment }\end{array}$ & $9(31 \%)$ & $5(28 \%)$ \\
$\begin{array}{l}\text { Cardiopulmonary failure } \\
\text { Multi-organ Failure }\end{array}$ & $6(21 \%)$ & $9(50 \%)$ \\
$\begin{array}{l}\text { Multiple Congenital } \\
\text { Anomalies }\end{array}$ & $6(31 \%)$ & $5(28 \%)$ \\
\hline
\end{tabular}

${ }^{\mathrm{a}}$ Note: Multiple indications allow for a sum over $100 \%$.

debriefing sheet were made available to staff in digital form on a NICU shared drive.

\section{Planning the study of the intervention}

Given the infrequency of CE events in the NICU, the nursing needs assessment survey was repeated after a 6-month period. Our team determined improvement targets for the primary outcomes based on results of needs assessment data: (1) increase percentage of nurse respondents' endorsement of "good" communication between nurses and the medical team by $15 \%$; (2) increase percentage of nurse respondents' subjective endorsement of ease of maintenance of patient comfort after CE by $15 \%$; and (3) increase frequency of post-event debrief by $50 \%$. A key driver diagram was created (Fig. 1). Outcomes of the initiative were studied using a time series design with 12 months baseline data preceding the intervention and 6 months of continuous monitoring after implementation of the checklist and debriefing sheet. Key process measures included: (1) respondents' ability to locate the checklist, (2) whether the checklist was used and reviewed with the medical team, (3) presence of a provider from the medical team near the bedside during and after CE to ensure adequate symptom management, and (4) perceived efficacy of the checklist and debriefing sheet in providing support to families and NICU staff. Data for these measures was collected in the post-event staff survey.

\section{Analysis}

Descriptive analysis was done for all measures, including pre- and post-intervention data. Process measure analysis was performed by construction of control charts, which tracked individual survey responses from post-event surveys over the three PDSA cycles.

\section{Ethical considerations}

This study was approved by the Institutional Review Board at Children's Hospital Los Angeles and was granted an exemption per Federal regulations 45 CFR 46.104[d][\#4(ii) and 4(iii)] and a waiver of HIPAA authorization per the privacy rule (45 CFR Part 160 and Subparts A and E of Part 164).

\section{Results}

\section{Characteristics of patients undergoing CE}

Out of 50 total deaths in the NICU during the initial 12month chart review, 58\% (29) occurred following CE. During the 6-month intervention period, $18 \mathrm{CE}$ events took place. Clinical characteristics of baseline and all postintervention CE events are presented in Table 1. The most 


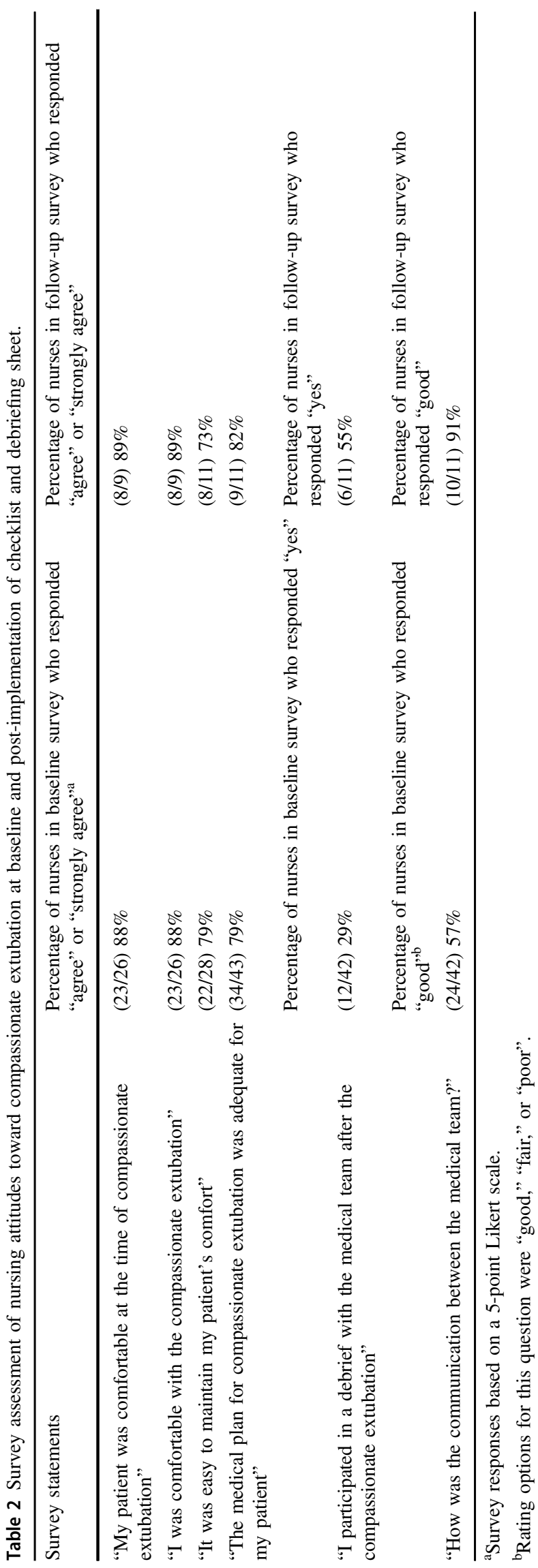

common indications for re-direction of care toward $\mathrm{CE}$ in the baseline group were severe neurologic impairment and multiorgan failure (both $31 \%, n=9$ ); cardiopulmonary failure was the primary indication for every $\mathrm{CE}$ event in the post-intervention group $(100 \%, n=9)$.

\section{Nursing attitudes toward CE}

Of the 291 NICU nurses invited to the baseline needs assessment survey, 43 nurses indicated that they had cared for a patient who underwent $\mathrm{CE}$ within the prior 12-month period at our institution. The post-intervention survey received 11 responses from 23 nurses identified as having been involved with a $\mathrm{CE}$ after checklist implementation; the response rate for post-intervention survey was $48 \%$. Table 2 compares nursing responses from baseline and post-intervention surveys. Differences in reported number of responses for different survey questions are due to survey responders leaving some survey questions unanswered. Notably, similar responses were reported at baseline and post-intervention for questions assessing perceived levels of patient comfort. Responses endorsing "good" communication with the medical team improved by $60 \%$, from $57 \%$ in baseline survey to $91 \%$ in postintervention survey. Reported rate of participation in a post-event debrief improved by $96 \%$, from a rate of $28 \%$ among baseline responders to $55 \%$ in post-intervention survey.

\section{Process measures}

85 post-event surveys were distributed to NICU staff members who cared for a patient undergoing CE. Of those 85 instances, we received 66 responses, with a response rate of $78 \%$. Among those responding, 82\% (54/66) reported using the checklist during a CE, and 68\% (45/66) reported reviewing the checklist with the medical team. The ability to locate the CE checklist varied by role: $83 \%$ of attendings $(15 / 18)$ and $91 \%$ fellows (10/11) were able to locate the checklist compared to only 59\% of nurses (10/17) and $46 \%$ of respiratory therapists $(6 / 13)$. There were 54 respondents who located and used the checklist, whose roles included: 14 attending neonatologists or hospitalists, 14 nurses, 11 neonatology fellows, 10 respiratory care practitioners, and 5 respondents who identified as "other," which included social workers and resident physicians rotating in the NICU. Of these 54 respondents, the percentage who indicated "agree" or "strongly agree" in response to survey questions assessing the impact of the checklist and debriefing sheet are shown in Table 3. Two control charts demonstrate improvement over time in percentage of survey respondents who agreed that the checklist was helpful for ensuring staff support during a CE event (Fig. 2a), and that the post-event 
Table 3 Post-event survey results.

Survey questions/statement

Percentage of respondents who indicated "Agree" or "Strongly Agree" $(n=54)$

"Was a provider from the medical team available and near bedside during and in the $(52 / 54) 96 \%$ short-term after the compassionate extubation to help ensure adequate symptom management?"

The medical team was effective in providing anticipatory guidance to families prior to compassionate extubation.

The checklist was helpful in ensuring family support during compassionate extubation.

The checklist was helpful in ensuring staff support during compassionate extubation.

The Post-Extubation Debriefing Sheet was helpful in ensuring team communication after compassionate extubation.

The Post-Extubation Debriefing Sheet was helpful in ensuring staff support after compassionate extubation.

$(43 / 54) 78 \%$

(40/54) $74 \%$

(43/54) $80 \%$

(30/54) $56 \%$

(30/54) $56 \%$ debriefing sheet was helpful in ensuring staff support after a CE event (Fig. 2b).

\section{Balance measures}

While the first goal of the medical team is to attend to the medical and emotional needs of these babies and their families, we recognize that NICU staff members, especially physicians and respiratory therapists, are often tasked with balancing the care of multiple complex patients. Given this reality, unmeasured but assumed balance measures included time spent by members of the medical team in debriefing as well as in checklist retrieval and completion, which have the potential to result in delays in care for other infants on the unit. These considerations are opportunities for future areas of study to examine how this affects the rest of the unit to ensure that the highest quality of care is provided for all NICU patients.

\section{Discussion}

\section{Summary}

CE occurs in a large percentage of NICU deaths and is a stressful, high-impact event for both families and staff. Our quality improvement project sought to improve staff communication and support during CE by implementing a checklist and debriefing sheet for the medical team. At 6 months post-intervention, nursing survey responses endorsing "good" communication with the medical team increased by $60 \%$, exceeding the project goal. The staff debrief rate following $\mathrm{CE}$ nearly doubled, also showing above-expected improvement. Subjective nursing assessments of patient comfort during and after $\mathrm{CE}$ found high ratings in both the baseline and post-intervention groups.

\section{Interpretation}

The CE checklist was developed with three goals in mind. The first goal was to provide the medical team with a standard framework offering considerations for both symptom management as well as family support during $\mathrm{CE}$ events. The second goal of the checklist was to facilitate clear and effective communication between members of the medical team caring for a patient undergoing $\mathrm{CE}$. The third goal was to prompt the medical team to conduct a postevent debrief after each CE. Importantly, the intention was not to prescribe medical management to the treating physician, and our finding of similarly high rates of positive response on subjective nursing assessments of patient comfort during and after CE indicates that there was appropriate symptom management in the majority of cases both pre and post-intervention. The majority of post-event respondents report reviewing the checklist with the medical team prior to $\mathrm{CE}$, confirming that it was successfully implemented. Our subsequent finding of improved nursing assessment of communication between the medical team post-implementation suggests that this improvement could be attributed to successful use of the checklist. Extrapolating from previous studies $[11,12,17,18]$, we expect that nurses who experience better communication with the medical team would have a better understanding of the care plan, and therefore provide improved care of the infant and communication with the family.

The checklist and debrief template were intended to work in tandem to prompt and then guide discussion during postevent staff debriefing. The rate of debrief following $\mathrm{CE}$ nearly doubled after these interventions, confirming that the interventions were successfully implemented. Furthermore, survey respondents endorsed that participation in the debrief was helpful for both team communication and staff emotional support, indicating that the debrief accomplished its intended goal. However, not all team members involved 

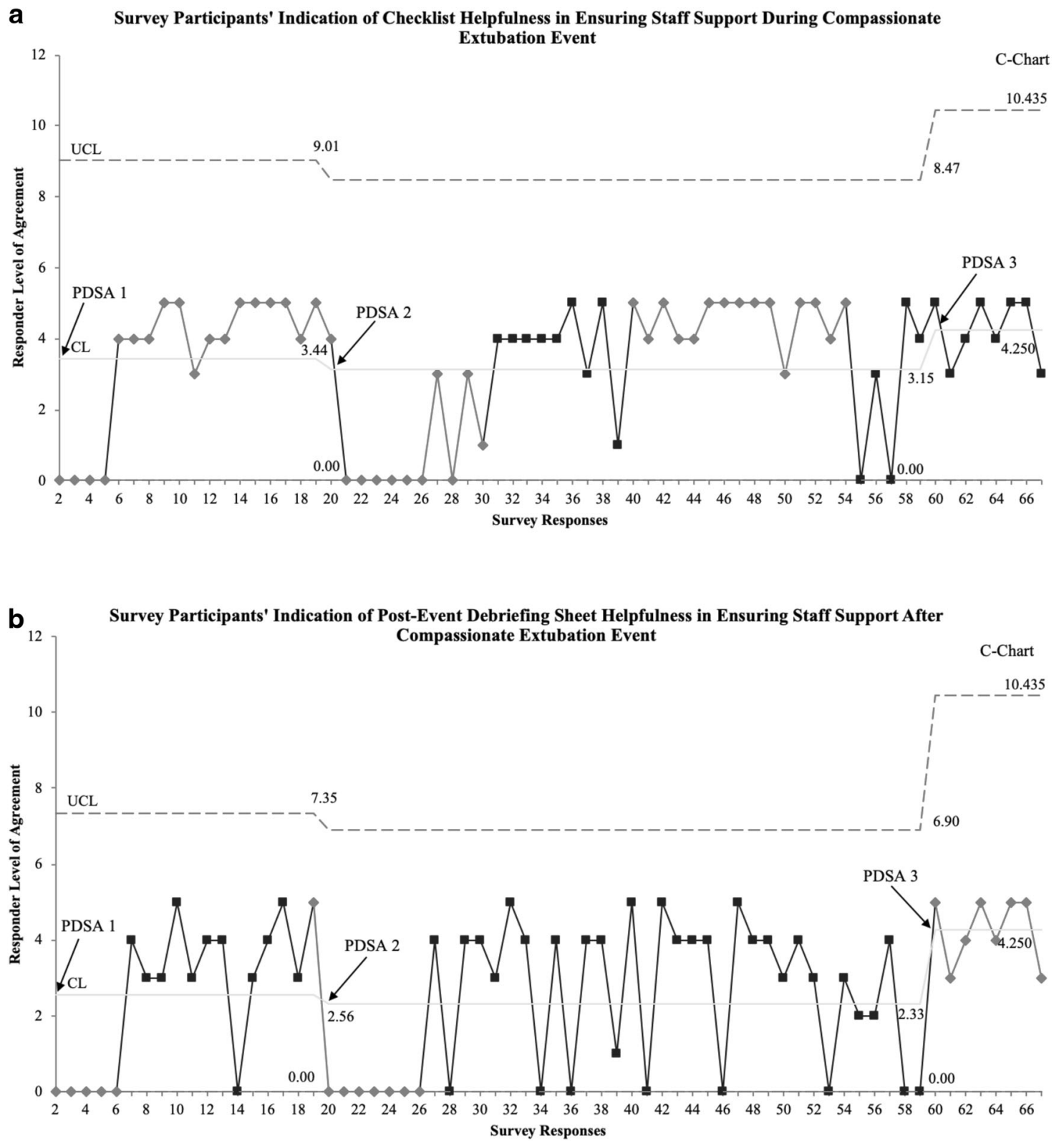

Fig. 2 Control charts for staff support during and after compassionate extubation. a Survey participants' indication of checklist helpfulness in ensuring staff support during compassionate extubation event. b Survey participants indication of post-event debriefing sheet helpfulness in ensuring staff support after compassionate extubation event. Level-of-agreement is rated on a 5-point Likert scale, with a response of 4 indicating "agree" and a response of 5 indicating "strongly agree." PDSA 1: checklist and debriefing sheet implementation. PDSA 2: checklist and debriefing sheet put together as a physical packet. PDSA 3: uploading checklist and debriefing sheet to a shared drive. Turquoise line indicates mean (CL). Red dotted line indicates upper control limit (UCL). with each CE had the opportunity to participate in the debrief event due to logistical constraints pertaining to extubations that occurred during or after shift changes. Consequently, staff members, such as primary nurses who may have had close relationships with the patient and family, could be most at risk for burnout and compassion fatigue. The opportunity for debrief should be expanded to offer all team members the opportunity to participate. This could be accomplished with the assistance of NICU social workers or nursing managers to identify team members in need of support and offer the opportunity for one-on-one debriefing.

Our similarly high rates of positive response on subjective nursing assessments of patient comfort during and after CE would indicate appropriate symptom management in the majority of cases both pre and post-intervention, suggesting that designating a provider to remain available during and after CE does not provide clear benefit in this 
regard. However, appointing a designated provider may improve communication between the bedside nurse and the medical team. This may have contributed to our finding of improved team communication post-implementation, although this contribution was not measured directly. Adaptation of the post-event survey to more specifically measure the effect of appointing a designated provider during CE could further clarify the extent of benefit.

\section{Limitations}

Our study was limited by a low response rate for the postintervention nursing survey, as fewer than half of nurses involved in post-intervention CE events ultimately completed the post-intervention survey, despite individual emails and in-person requests for survey participation. Barriers to checklist use were more frequently reported among nurses and respiratory therapists than physicians. The most common barriers were lack of awareness and inability to locate the checklist. These warrant targeted interventions to improve NICU staff awareness in future PDSA cycles. One additional limitation of this study is that parent experiences during $\mathrm{CE}$ were not directly measured, although there is known correlation between nurse comfort and improved parent experience during end-of-life care [10].

In addition to improving staff awareness and survey response, implementation of educational sessions for NICU staff has been targeted as another possible subsequent intervention. Sessions on neonatal end-of-life anticipatory guidance, symptom management, and strategies to combat compassion fatigue could serve as interventions in future PDSA cycles to improve staff preparedness during CE's. We feel it is particularly important to give special consideration to training physicians, who are tasked with leading the medical team during these challenging events. Further targets could also include implementation of this projects' checklist, debriefing template, and end-of-life education in other departments in the hospital where CEs occur.

\section{Conclusions}

Our project describes one institution's practices and nursing attitudes toward $\mathrm{CE}$ in the neonatal intensive care unit (NICU), as well as the implementation of a novel pilot protocol for standardization of CE. Implementation of a checklist and post-event team debriefing sheet was associated with an increase in the rate of post-event team debriefs and an improved assessment of team communication. These improvements with $\mathrm{CE}$ and end-of-life care move us closer to our ultimate goal of providing the most compassionate, skillful care possible to every patient and family in our NICU.

Acknowledgements We would like to acknowledge the support of the divisions of Neonatology and Comfort and Palliative Care at Children's Hospital Los Angeles.

Author contributions Dr KD conceptualized and designed the study, designed the data collection instruments, collected data, performed the analyses, drafted the initial paper, and reviewed and revised the paper. Ms MF and Dr SD conceptualized and designed the study, designed the data collection instruments, and reviewed and revised the paper. Ms MC designed the data collection instruments, collected data, performed the analyses, drafted part of the initial paper, and reviewed and revised the paper. Drs SN and DL conceptualized and designed the study, coordinated and supervised data collection, and critically reviewed the paper for important intellectual content. All authors approved the final paper as submitted and agree to be accountable for all aspects of the work.

Funding No funding was secured for this study.

\section{Compliance with ethical standards}

Conflict of interest The authors declare no competing interests.

Publisher's note Springer Nature remains neutral with regard to jurisdictional claims in published maps and institutional affiliations.

\section{References}

1. Michel MC, Colaizy TT, Klein JM, Segar JL, Bell EF. Causes and circumstances of death in a neonatal unit over 20 years. Pediatr Res. 2018;83:829-33.

2. Wilkinson DJ, Fitzsimons JJ, Dargaville PA, Campbell NT, Loughnan PM, McDougall PN, et al. Death in the neonatal intensive care unit: changing patterns of end of life care over two decades. Arch Dis Child Fetal Neonatal Ed. 2006;91:F268-71.

3. Weiner J, Sharma J, Lantos J, Kilbride H. How infants die in the neonatal intensive care unit: trends from 1999 through 2008. Arch Pediatr Adolesc Med. 2011:165:630-4.

4. Gibson K, Hofmeyer A, Warland J. Nurses Providing End-of-Life Care for Infants and Their Families in the NICU: a Review of the Literature. Adv Neonatal Care. 2018;18:471-9.

5. Hall SL, Cross J, Selix NW, Patterson C, Segre L, Chuffo-Siewert $\mathrm{R}$, et al. Recommendations for enhancing psychosocial support of NICU parents through staff education and support. J Perinatol. 2015;35:S29-36.

6. Gold KJ. Navigating care after a baby dies: a systematic review of parent experiences with health providers. J Perinatol. 2007;27: $230-7$.

7. Meert KL, Thurston CS, Thomas R. Parental coping and bereavement outcome after the death of a child in the pediatric intensive care unit. Pediatr Crit Care Med. 2001;2:324-8.

8. Ahern K. What neonatal intensive care nurses need to know about neonatal palliative care. Adv Neonatal Care. 2013;13:108-14.

9. Peng NH, Chen CH, Huang LC, Liu HL, Lee MC, Sheng CC. The educational needs of neonatal nurses regarding neonatal palliative care. Nurse Educ Today. 2013;33:1506-10.

10. Brooten D, Youngblut JM, Seagrave L, Caicedo C, Hawthorne D, Hidalgo I, et al. Parent's perceptions of health care providers actions around child ICU death: what helped, what did not. Am J Hosp Palliat Care. 2013;30:40-9. 
11. Dombrecht L, Cohen J, Cools F, Deliens L, Goossens L, Naulaers $\mathrm{G}$, et al. Psychological support in end-of-life decision-making in neonatal intensive care units: full population survey among neonatologists and neonatal nurses. Palliat Med. 2020;34:430-4.

12. Bloomer MJ, O'Connor M, Copnell B, Endacott R. Nursing care for the families of the dying child/infant in paediatric and neonatal ICU: nurses' emotional talk and sources of discomfort. A mixed methods study. Aust Crit Care. 2015;28:87-92.

13. Winters BD, Gurses AP, Lehmann H, Sexton JB, Rampersad CJ, Pronovost PJ. Clinical review: checklists - translating evidence into practice. Crit Care. 2009;13:210.

14. Lewis SL. Exploring NICU Nurses' Affective Responses to Endof-Life Care. Adv Neonatal Care. 2017;17:96-105.
15. Riotte CO, Kukora SK, Keefer PM, Firn JI. Identifying the Types of Support Needed by Interprofessional Teams Providing Pediatric End-of-Life Care: a Thematic Analysis. J Palliat Med. 2018;21:422-7.

16. Courtlandt CD, Noonan L, Feld LG. Model for improvement Part 1: a framework for health care quality. Pediatr Clin North Am. 2009;56:757-78.

17. Kopelman AE. Understanding, avoiding, and resolving end-of-life conflicts in the NICU. Mt Sinai J Med. 2006;73:580-6.

18. Kowalski WJ, Leef KH, Mackley A, Spear ML, Paul DA. Communicating with parents of premature infants: who is the informant? J Perinatol. 2006;26:44-8. 